\title{
Electron pairing by Coulomb repulsion in narrow band structures
}

\author{
Klaus M. Frahm $\odot$ and Dima L. Shepelyansky $\odot$ \\ Laboratoire de Physique Théorique, IRSAMC, Université de Toulouse, CNRS, UPS, 31062 Toulouse, France
}

(Received 16 February 2020; revised manuscript received 2 May 2020; accepted 3 June 2020; published 17 June 2020)

\begin{abstract}
We study analytically and numerically dynamics and eigenstates of two electrons with Coulomb repulsion on a tight-binding lattice in one and two dimensions. The total energy and momentum of electrons are conserved and we show that for a certain momentum range the dynamics is exactly reduced to an evolution in an effective narrow energy band where the energy conservation forces the two electrons to propagate together through the whole system at moderate or even weak repulsion strength. We argue that such a mechanism of electron pair formation by the repulsive Coulomb interaction is rather generic and that it can be at the origin of unconventional superconductivity in twisted bilayer graphene.
\end{abstract}

DOI: 10.1103/PhysRevResearch.2.023354

\section{INTRODUCTION}

The interactions of electrons in narrow band structures play an important role in various physical processes. Often the theoretical analysis is done in the framework of electrons on a lattice with short-range on-site interactions as described in works of Gutzwiller [1,2] and Hubbard [3]. Recently, the interest in narrow band structures with strong Coulomb electron-electron interactions has been inspired by the observation of unconventional superconductivity in magic-angle twisted bilayer graphene (MATBG) [4]. Such structures are characterized by a very high ratio of the critical temperature of the superconducting transition to the Fermi energy $T_{c} / E_{F}$ [4] and complex phase diagrams of superconducting and insulating phases $[5,6]$. Experimental results for MATBG clearly show the importance of long-range Coulomb electronelectron interactions in these structures [4-7]. From the theoretical side it has been shown that for small twisted angles the moiré pattern leads to a formation of a superlattice with a unit cell containing more than 10,000 atoms that significantly modifies the low-energy structure. Extensive numerical studies by quantum-chemistry methods show the appearance of flat lowest-energy minibands [8-11]. These bands are rather narrow and thus the Coulomb interactions play an important role as pointed out in early theoretical studies [12]. The existence of narrow flat bands was also confirmed in recent MATBG experiments [13].

In this work we show that the narrow band structure of the free-electron spectrum leads to a number of unusual properties of their propagation in the presence of strong, moderate, or even weak Coulomb repulsion between electrons. We discuss these properties for a model of two electrons with

Published by the American Physical Society under the terms of the Creative Commons Attribution 4.0 International license. Further distribution of this work must maintain attribution to the author(s) and the published article's title, journal citation, and DOI.
Coulomb interaction propagating on a standard tight-binding lattice considered in [1-3]. For the simple case of shortrange Hubbard-type interactions it was shown [3,14] that the quantum problem can be mapped to an implicit equation for the energy eigenvalues, allowing one to identify individual bound pair states at strong interaction values. However, in the case of long-range Coulomb interactions, this mathematical simplification is not possible and the physical situation, especially in two dimensions with potentially chaotic dynamics in the relative coordinate, is more complicated. Our results take these complications into account and show the appearance of pairing of two electrons induced even by a moderate Coulomb repulsion with ballistic pairs propagating over the whole system size. Below we describe the physical properties of such pairs of two interacting particles (TIPs). Furthermore, we show that this effect strongly depends on the values of the conserved center of mass momenta.

\section{QUANTUM TIGHT-BINDING MODEL OF TWO ELECTRONS}

The quantum Hamiltonian of the model in $d=1$ or 2 dimensions has the standard form [1-3]

$$
H=-\sum_{\langle j, l\rangle}|j\rangle\left\langle l\left|+\sum_{j} \frac{U}{1+r(j)}\right| j\right\rangle\langle j|,
$$

where $j=\left(x_{1}, x_{2}\right)\left[j=\left(x_{1}, x_{2}, y_{1}, y_{2}\right)\right]$ is a multi-index for $d=1 \quad(d=2)$; each index variable takes values $x_{1}, x_{2}, y_{1}, y_{2} \in\{0, \ldots, N-1\}$, with $N$ the linear system size with periodic boundary conditions. The first sum in (1) describes the electron hopping between nearby sites on a onedimensional (1D) (or 2D square) lattice with a hopping amplitude taken as the energy unit. The second sum in (1) represents a (regularized) Coulomb-type long-range interaction with amplitude $U$ and the distance $r(j)$ between two electrons. For one dimension we have, due to the periodic boundary conditions, $r(j)=\Delta \bar{x}$ with $\Delta \bar{x}=\min (\Delta x, N-\Delta x)$ and relative coordinate $\Delta x=x_{2}-x_{1}$, which is taken modulo $N$ (i.e., $\Delta x=$ 
$x_{2}-x_{1}+N$ for $\left.x_{2}-x_{1}<0\right)$. For two dimensions we have $r(j)=\sqrt{\Delta \bar{x}^{2}+\Delta \bar{y}^{2}}$. Furthermore, we consider symmetric (spatial) wave functions with respect to particle exchange, assuming an antisymmetric spin-singlet state (similar results are obtained for antisymmetric wave functions). In the absence of interactions at $U=0$ the spectrum of free electrons has the standard band structure $E=-2 \sum_{\mu=1,2 ; \alpha} \cos p_{\mu \alpha}$, with $\mu=1,2$ the electron index and $\alpha=x$ in one dimension $(\alpha \in$ $\{x, y\}$ in two dimensions) the index for each spatial dimension. With periodic boundary conditions, each momentum $p_{\mu \alpha}$ is an integer multiple of $2 \pi / N$.

\section{CLASSICAL DYNAMICS OF ELECTRON PAIRS}

The corresponding classical dynamics in two dimensions is described by the Hamiltonian

$$
H=-2 \sum_{\mu=1,2 ; \alpha \in\{x, y\}} \cos p_{\mu \alpha}+U_{C}\left(x_{1}, x_{2}, y_{1}, y_{2}\right),
$$

with $U_{C}=U /\left[1+\sqrt{\left(x_{1}-x_{2}\right)^{2}+\left(y_{1}-y_{2}\right)^{2}}\right]$ and conjugated variables of momentum $p_{\mu x}$ and $p_{\mu y}$ and coordinates $x_{\mu}$ and $y_{\mu}$ [in one dimension we have in (2) only $p_{\mu x}$ and $x_{\mu}$ ]. In one dimension there are two integrals of motion, with the total energy $E=H$ and total momentum $p_{+}=p_{1 x}+p_{2 x}$ leading to integrable TIP dynamics. In two dimensions we have three integrals of motion $E, p_{+x}$, and $p_{+y}$ for four degrees of freedom and therefore the dynamics of the two electrons is generally chaotic, as it is shown in Fig. S1 of [15], Sec. S1.

Writing $\cos \left(p_{1 x}\right)+\cos \left(p_{2 x}\right)=2 \cos \left(p_{+x} / 2\right) \cos \left[\left(p_{2 x}-\right.\right.$ $\left.p_{1 x}\right) / 2$ ] (and similarly for $y$ ), we see that at given values of $p_{+x}$ and $p_{+y}$ the kinetic energy is bounded by $\Delta E=4 \sum_{\alpha}\left|\cos \left(p_{+\alpha} / 2\right)\right|$. Therefore, for TIP states with $E>\Delta E$, the two electrons cannot separate and they propagate as one pair. In particular, for $p_{+x}=p_{+y}=\pi+\delta$ (with $|\delta| \ll 1$ ) close to $\pi$, there are compact Coulomb electron pairs even for very small interactions $U$ as soon as $\Delta E \approx 2 d|\delta|<U \ll B_{d}$, with $B_{d}=8 d+U$ the maximal energy bandwidth in $d$ dimensions. The center of mass velocity of such pairs [in direction $\alpha \in\{x, y\}$ ] is $v_{+\alpha}=$ $\left(v_{1 \alpha}+v_{2 \alpha}\right) / 2=2 \cos (\delta / 2) \sin \left(p_{1 \alpha}-\delta / 2\right) \approx 2 \sin p_{1 \alpha}$ and it may be close to a maximal velocity $v_{+\alpha}=2$. Figure $\mathrm{S} 1$ of [15] clearly confirms the pair formation at small $U$ values and the pair propagation through the whole system.

\section{QUANTUM TIP EIGENSTATES AND DYNAMICS IN ONE AND TWO DIMENSIONS}

The factor $2 \cos \left(p_{+} / 2\right)$ for the kinetic energy (in one dimension) at a given value of $p_{+}$can also be obtained from the quantum model. Using a suitable unitary transformation (see [15], Sec. S2), one can transform the quantum Hamiltonian (1) to a block diagonal form where the different blocks on the diagonal correspond, for each value of the conserved quantum number $p_{+}$, to an effective one-particle tight-binding model (in $\Delta x$ space) with nearest-neighbor hopping matrix element $-2 \cos \left(p_{+} / 2\right)$ and a diagonal potential given by $U /(1+\Delta \bar{x})$ (see [15], Sec. S2 for details, in particular the boundary conditions and the generalization to the 2D case). Physically, this corresponds to a single particle in $\Delta x$ space with a
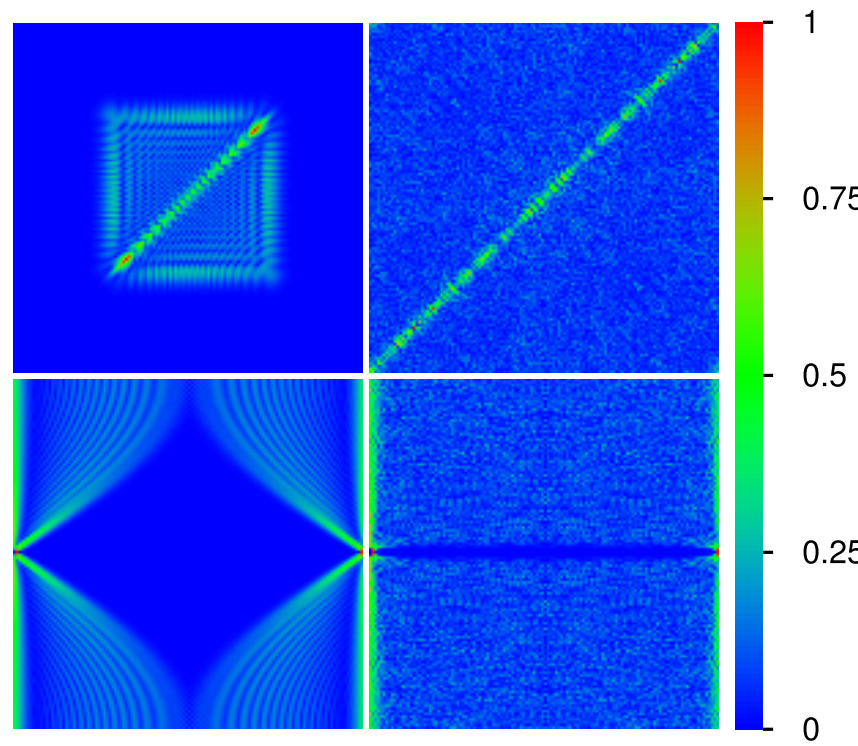

FIG. 1. Plot of wave function amplitudes obtained from the exact quantum time evolution in one dimension at times $t=138 \Delta t$ and $10^{5} \Delta t$ in left and right panels, respectively $\left(\Delta t=1 / B_{1}\right.$ is an elementary time step of inverse bandwidth $B_{1}=8+U$ ). The top panels show the TIP wave function amplitude $\left|\psi\left(x_{1}, x_{2}, t\right)\right|$ at $x_{1}$ and $x_{2}$ for both axes. The bottom panels show $\left|\bar{\psi}\left(p_{+}, \Delta x, t\right)\right|$ with $\Delta x=$ $x_{2}-x_{1}$ (taken modulo $N$ ) the relative coordinate (horizontal axis) and $0 \leqslant p_{+}<2 \pi$ the total momentum (vertical axis). The initial state at $t=0$ is a symmetrized state with one electron localized at $N / 2$ and the other one at $N / 2+1$, i.e., initial distance $\Delta \bar{x}=1$. The other parameters are $U=1$ and $N=128$. The color bar represents (here and in certain subsequent figures) the ratio of the quantity shown (here the modulus of the wave function amplitude) to its maximal value. Related videos are available in $[15,16]$.

kinetic energy rescaled by $2 \cos \left(p_{+} / 2\right)$ and moving in a given potential with a maximal value $U$ for $\Delta x$ close to 0 or $N$.

The eigenstates can be efficiently calculated for large system sizes since each block for a given value of $p_{+}$can be diagonalized individually. Also the quantum time evolution (for $\hbar=1$ ) can be efficiently computed (up to $N=1024$ ) by transforming the initial state $\psi\left(x_{1}, x_{2}\right)$ into a block representation $\bar{\psi}\left(p_{+}, \Delta x\right)$ and computing the time evolution exactly in each $p_{+}$sector using the exact block eigenstates (details in [15], Sec. S2).

A typical example of the TIP time evolution in one dimension is shown in the top panels of Fig. 1 for the initial electron positions (localized at $x_{\mu} \approx N / 2$ ) at a distance $R=\Delta \bar{x}=1$ for $U=1$ and $N=128$. We see a wave front of free propagating separated electrons (square at short times in the top left panel) and at the same time free propagation of the Coulomb electron pair along the diagonal $x_{1}=x_{2}$. At long times the density for separated electrons is uniformly distributed over the whole system, while the density for Coulomb pairs is homogeneously distributed over the whole diagonal, keeping a relatively small pair size (top right panel). The bottom panels show the block representation $\left|\bar{\psi}\left(p_{+}, \Delta x, t\right)\right|$ of the same states as in the top panels. In this representation the initial state corresponds to two red vertical lines at $\Delta x=1$ and $\Delta x=$ $N-1$ for perfect localization at these values and a uniform 

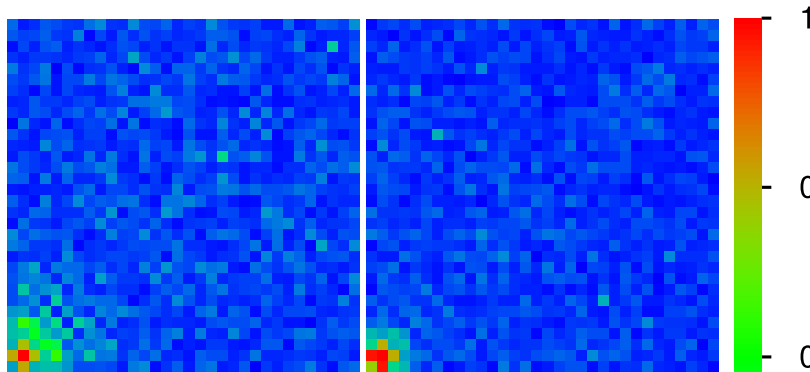

1
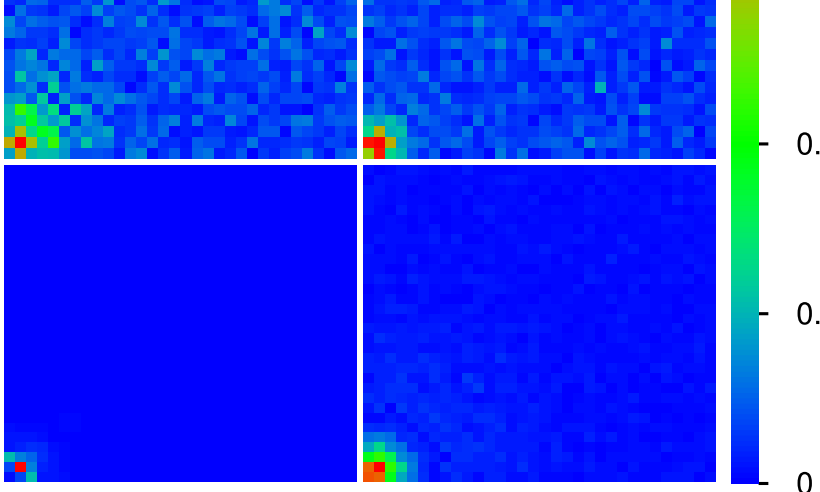

0.5

FIG. 2. Plot of wave function amplitude $\left|\bar{\psi}\left(p_{+x}, p_{+y}, \Delta x, \Delta y\right)\right|$ in block representation obtained from the 2D quantum time evolution at iteration time $t=10^{5} \Delta t$ in the $\Delta x-\Delta y$ plane for certain $p_{+x}$ and $p_{+y}$ sectors and $U=2\left[\Delta t=1 / B_{2}=1 /(16+U)\right]$. The initial block state at $t=0$ is localized at $\Delta x=\Delta y=1$. All panels show a closeup of the region $(0 \leqslant \Delta x, \Delta y<32)$. The values of $p_{+}=p_{+x}=p_{+y}$ and $N$ are $p_{+}=0$ and $N=128$ (top left), $p_{+}=21 \pi / 32 \approx 2 \pi / 3$ and $N=128$ (top right), $p_{+}=63 \pi / 64 \approx \pi$ and $N=128$ (bottom left), and $k=85 \pi / 128 \approx 2 \pi / 3$ and $N=512$ (bottom right). Related videos are available in $[15,16]$.

distribution in the $p_{+}$direction. With increasing time a part of the density stays quite well localized at the initial values with some modest increase of the initial width. The other fraction of the density propagates horizontally and becomes roughly uniform at sufficiently long times. The speed of the horizontal propagation is apparently proportional to $\cos \left(p_{+} / 2\right)$ and for $p_{+} \approx \pi$ there is actually a small regime of strong, nearly perfect localization, where the weight of the propagating density is close to 0 . In this case the kinetic energy can be considered as a small perturbation of the interaction due to the small ratio $2\left|\cos \left(p_{+} / 2\right)\right| / U \ll 1$. However, even for larger values of this ratio there is still a considerable fraction of the density that stays localized.

Further results of the 1D quantum case are given in Figs. S2-S5 of [15], Sec. S3, including an analysis of the Husimi functions (see, e.g., [17,18]).

In order to access larger system sizes up to $N=512$ we compute, for the 2D case, the exact quantum time evolution only in individual sectors with $p_{+x}=p_{+y}$ (and exploiting certain symmetries [15]). The initial state is localized at $\Delta \bar{x}=\Delta \bar{y}=1$ but also at the chosen value of $p_{+x}=p_{+y}$. This corresponds to a wave in the center of mass direction with momenta $p_{+x}$ and $p_{+y}$, which is however perfectly localized in the relative coordinate.

Figure 2 clearly shows that there is a considerable probability that both electrons stay together even at very long times. There is however, for $p_{+x, y}=0$ or $p_{+x, y} \approx 2 \pi / 3$, also a certain complementary density for free-electron propagation which becomes less visible for larger $N=512$. For $p_{+x, y} \approx \pi$ there is actually nearly perfect localization even at $N=128$.
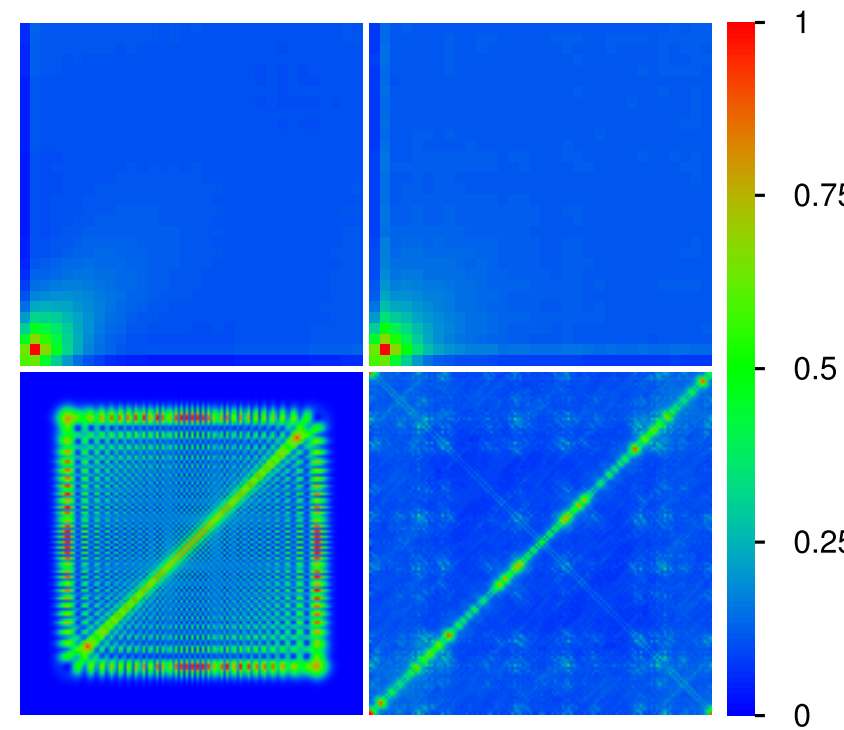

1

FIG. 3. Two-dimensional wave function densities obtained from the time evolution shown at times $t=445 \Delta t$ and $10^{4} \Delta t$ in left and right panels, respectively, for initial electron positions at approximately $(N / 2, N / 2)$, with $N=128$ and $U=2\left[\Delta t=1 / B_{2}=\right.$ $1 /(16+U)$ is the Trotter integration time step]. The top panels show a close-up of the density for $(0 \leqslant \Delta x, \Delta y<32)$ in the $\Delta x-\Delta y$ plane of relative coordinates obtained from a sum over $x_{1}$ and $y_{1}$. The bottom panels show the density in the $x_{1}-x_{2}$ plane obtained from a sum over $y_{1}$ and $y_{2}$. The corresponding values of the probability near the diagonal $w_{10}$ are $w_{10}=0.106$ and 0.133 for the left and right panels, respectively (see the text). Related videos are available in $[15,16]$.

This confirms the formation of Coulomb electron pairs in the 2D quantum case and as in one dimension also the particularly strong localization in relative coordinates for sectors with $p_{+x, y} \approx \pi$.

We also computed the full space 2D quantum time evolution using the Trotter formula approximation (see, e.g., [19] for computational details) with a Trotter time step $\Delta t=$ $1 / B_{2}=1 /(16+U)=1 / 18$ for $U=2$ and $N=128$. Here we choose an initial condition with both particles localized at approximately $(N / 2, N / 2)$ such that $\Delta \bar{x}=\Delta \bar{y}=1$. The results of Fig. 3 clearly show that there is a significant probability of electron pair formation with a propagation of pairs over the whole system (high probability of $\Delta x$ and $\Delta y$ at values close to zero in the top panels and near the diagonal $x_{1} \approx x_{2}$ in the bottom panels). At the same time there is also a certain probability to have practically independent electrons with ballistic propagation through the lattice at moderate times (bottom left panel) and an approximate homogeneous distribution over the whole system at long times (bottom right panel).

In order to characterize the pair formation probability we compute the quantum probability $w_{10}(t)$ to find both electrons at a finite distance from each other $\Delta \bar{x} \leqslant \Delta R=10$ and $\Delta \bar{y} \leqslant$ $\Delta R$. At long times we find that the pair formation probability is $w_{10}=0.133$, while the probability to have independent electrons is $1-w_{10}$.

The time dependence of the pair formation probability $w_{10}$ for both types of $2 \mathrm{D}$ quantum time evolution is 


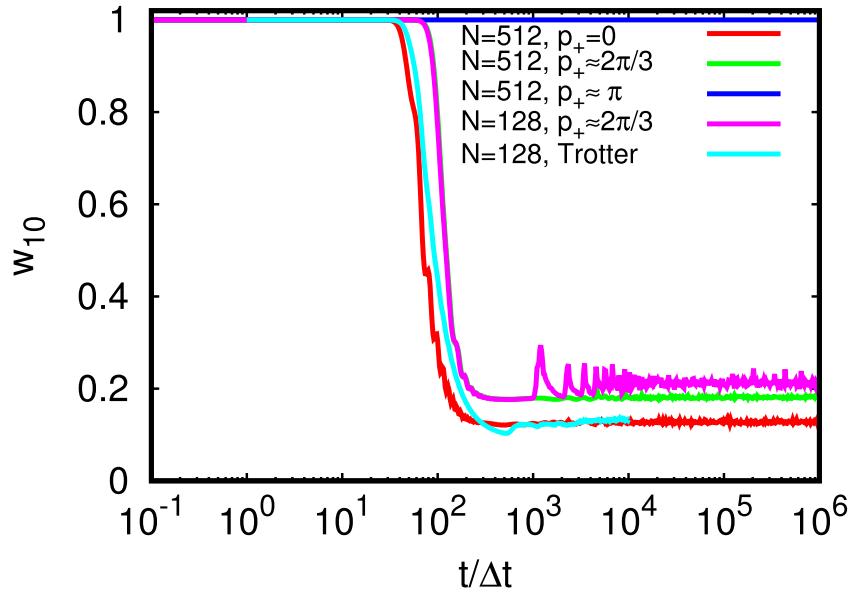

FIG. 4. Time dependence of the pair formation probability $w_{10}$ for $U=2$ and different cases of either the exact 2D time evolution in certain sectors of conserved momenta $p_{+}=p_{+x}=p_{+y}$ or the full space 2D time evolution using the Trotter formula approximation (initial states as in Figs. 2 and 3). The precise values of $p_{+}$are $p_{+}=0, p_{+}=85 \pi / 128 \approx 2 \pi / 3$, and $p_{+}=255 \pi / 256 \approx \pi$ for $N=$ 512 and $p_{+}=21 \pi / 32 \approx 2 \pi / 3$ for $N=128$. The top blue line at $w_{10}=1$ is accurate with a numerical error below $10^{-14}$ for all time values.

shown in Fig. 4 for $U=2$ and $N \in\{128,512\}$. For $p_{+} \approx$ $\pi$ we have $w_{10}=1$ with numerical accuracy, while for $p_{+} \approx 2 \pi / 3$ and $p_{+}=0$ this probability decreases with time but saturates at rather high values of $w_{10} \approx 0.2$ and 0.13 , respectively. The saturation value $w_{10} \approx 0.133$ for the full space Trotter formula approximation is very close to the case $p_{+}=0$.

The initial state with the pair momentum $p_{+}$approximately corresponds to the electron Fermi energy $E_{F} \approx$ $-4 \cos \left(p_{+} / 2\right)$, assuming a relatively moderate or weak interaction $U \ll B_{d}$. Thus the variation of $p_{+}$in the range $0 \leqslant p_{+} \leqslant 2 \pi$ corresponds to the variation of the filling factor $v$ in the range $0 \leqslant v \leqslant 1$ with $v=\left[1-\cos \left(p_{+} / 2\right)\right] / 2$. The dependence $w_{10}(v)$ on $v$ is shown in Fig. 5 for $U=0.5,2$ and $N=256$ (the data for $N=512$ are very close). We see that for small $v \ll 1 / 2$ the electron pair formation probability is relatively small, but for $1 / 3 \leqslant v \leqslant 1 / 2$ it takes, for $U=2$ $(U=0.5)$, values from $w_{10}=0.17\left(w_{10}=0.11\right)$ to $w_{10}=$ 1. The values of $w_{10}$ are significantly above the ergodic probability $w_{\text {erg }}=(21 / N)^{2} \approx 0.0067$ for $N=256$, assuming a uniform distribution.

More results for the quantum time evolution and eigenstates properties in two dimensions are given in [15] (see Figs. S6-S11). In particular, there is a clear scaling dependence of $w_{10}$ (and other related characteristics) on the ratio $2 \cos \left(p_{+} / 2\right) / U$ (Figs. S10 and S11 of [15])

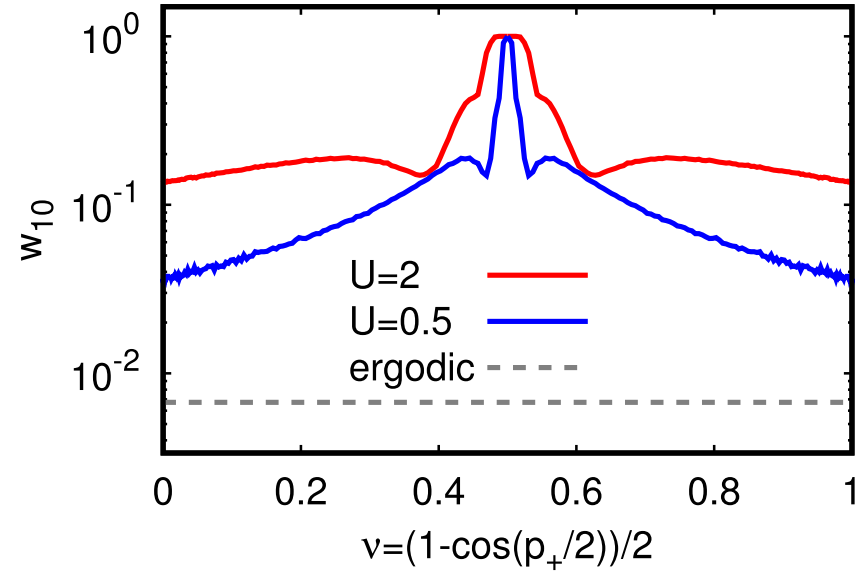

FIG. 5. Dependence of the pair formation probability $w_{10}$ on the filling factor $v$ for $U=2$ (red curve) and 0.5 (blue curve) for $N=256$ in two dimensions. The dashed line shows the ergodic probability $w_{\text {erg }}$ assuming a uniform wave function.

\section{DISCUSSION}

The presented analytical and numerical analysis definitely shows that a specific energy dispersion law of free electrons in narrow bands leads to the possibility of pair formation induced by Coulomb repulsion between electrons. The pairing of electrons already exists for a relatively moderate, or even small, repulsion strength $U$. Of course, this analysis is performed in the framework of only two interacting electrons. However, we conjecture that these Coulomb electron pairs will still exist even at finite electron density $v$. Indeed, a propagating pair can feel interactions from other electrons as a certain mean field potential which should not destroy pairs with a relatively strong coupling. In the presence of an external space inhomogeneous potential the independent electrons can be localized by the potential, while the pairs, protected by their effective coupling energy $\Delta_{c} \sim U / \Delta R$, can remain insensitive to the potential propagating through the whole system (we assume that $\Delta R$ is of the order of several lattice units as for $w_{10}$ computations). Thus such pairs can form a condensate leading to the emergence of a superconducting state. We argued that the emergence of Coulomb electron pairs in narrow band structures can be at the origin of superconductivity in MATBG experiments.

\section{ACKNOWLEDGMENTS}

This work was supported in part by the Programme Investissements d'Avenir through Grants No. ANR-11-IDEX0002-02 and No. ANR-10-LABX-0037-NEXT (project THETRACOM). This work was granted access to the HPC resources of CALMIP (Toulouse) under Grant No. 2019P0110.
[1] M. C. Gutzwiller, Effect of Correlation on the Ferromagnetism of Transition Metals, Phys. Rev. Lett. 10, 159 (1963).

[2] M. C. Gutzwiller, Correlation of electrons in a narrow $s$ band, Phys. Rev. 137, A1726 (1965).
[3] J. Hubbard, Electron correlations in narrow energy bands, Proc. R. Soc. London Ser. A 276, 238 (1963).

[4] Y. Cao, V. Fatemi, S. Fang, K. Watanabe, T. Taniguchi, E. Kaxiras, and P. Jarillo-Herrero, Unconventional 
superconductivity in magic-angle graphene superlattices, Nature (London) 556, 43 (2018).

[5] Y. Cao, V. Fatemi, A. Demir, S. Fang, S. L. Tomarken, J. Y. Luo, J. D. Sanchez-Yamagishi, K. Watanabe, T. Taniguchi, E. Kaxiras, R. C. Ashoori, and P. Jarillo-Herrero, Correlated insulator behavior at half-filling in magic-angle graphene superlattices, Nature (London) 556, 80 (2018).

[6] X. Lu, P. Stepanov, W. Yang, M. Xie, M. A. Aamir, I. Das, C. Urgell, K. Watanabe, T. Taniguchi, G. Zhang, A. Bachtold, A. H. MacDonald, and D. K. Efetov, Superconductors, orbital magnets, and correlated states in magic angle bilayer graphene, Nature (London) 574, 653 (2019).

[7] P. Stepanov, I. Das, X. Lu, A. Fahimniya, K. Watanabe, T. Taniguchi, F. H. L. Koppens, K. Lischner, L. Levitov, and D. K. Efetov, The interplay of insulating and superconducting orders in magic-angle graphene bilayers, arXiv:1911.09198.

[8] M. Koshino, N. F. Q. Yuan, T. Koretsune, M. Ochi, K. Kuroki, and L. Fu, Maximally Localized Wannier Orbitals and the Extended Hubbard Model for Twisted Bilayer Graphene, Phys. Rev. X 8, 031087 (2018).

[9] N. F. Q. Yuan and L. Fu, Model for the metal-insulator transition in graphene superlattices and beyond, Phys. Rev. B 98, 045103 (2018).

[10] J. Kang and O. Vafek, Symmetry, Maximally Localized Wannier States, and a Low-Energy Model for Twisted Bilayer Graphene Narrow Bands, Phys. Rev. X 8, 031088 (2018).

[11] X. Wu, W. Hanke, M. Fink, M. Klett, and R. Thomale, Harmonic fingerprint of unconventional superconductivity in twisted bilayer graphene, Phys. Rev. B 101, 134517 (2020).
[12] R. Bistrizer and A. H. MacDonald, Moire bands in twisted double-layer graphene, Proc. Natl. Acad. Sci. USA 108, 12233 (2011).

[13] M. I. B. Utama, R. J. Koch, K. Lee, N. Leconte, H. Li, S. Zhao, L. Jiang, J. Zhu, K. Watanabe, T. Taniguchi, P. D. Ashby, A. Weber-Bargioni, A. Zettl, C. Jozwiak, J. Jung, E. Rotenberg, A. Bostwick, and F. Wang, Visualization of the flat electronic band in twisted bilayer graphene near the magic angle twist, arXiv:1912.00587.

[14] S. M. Mahajan and A. Thyagaraja, Exact two-body bound states with Coulomb repulsion in a periodic potential, J. Phys. A: Math. Gen. 39, L667 (2006).

[15] See Supplemental Material at http://link.aps.org/supplemental/ 10.1103/PhysRevResearch.2.023354 that contains mathematical details on the numerical diagonalization and quantum time evolution methods exploiting the conserved total momentum and extra figures and videos that support the main conclusions and results.

[16] K. M. Frahm and D. L. Shepelyansky, available at http: //www.quantware.ups-tlse.fr/QWLIB/coulombelectronpairs/ (accessed January 2020).

[17] S.-J. Chang and K.-J. Shi, Evolution and exact eigenstates of a resonant quantum system, Phys. Rev. A 34, 7 (1986).

[18] K. M. Frahm, R. Fleckinger, and D. L. Shepelyansky, Quantum chaos and random matrix theory for fidelity decay in quantum computations with static imperfections, Eur. Phys. J. D 29, 139 (2004).

[19] K. M. Frahm and D. L. Shepelyansky, Delocalization of two interacting particles in the 2D Harper model, Eur. Phys. J. B 89, 8 (2016). 